\title{
Image Sensor Noise Profiling by Voting Based Curve Fitting
}

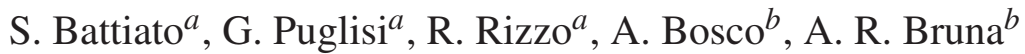 \\ ${ }^{a}$ University of Catania, Viale A. Doria, Catania, Italy \\ Image Processing Lab \\ http://iplab.dmi.unict.it \\ ${ }^{b}$ AST - Computer Vision, STMicroelectronics Catania, Italy
}

\begin{abstract}
The output quality of an image filter for reducing noise without damaging the underlying signal, strongly depends on the accuracy of the noise model in characterizing the noise introduced by the acquisition device. In this paper we provide a solution for characterizing signal dependent noise injected at shot time by the image sensor. Different fitting models describing the behavior of noise samples are analyzed, with the aim of finding a model that offers the most accurate coverage of the sensor noise under any of its operating conditions. The noise fitting equation minimizing the residual error is then identified. Moreover, a novel algorithm able to obtain the noise profile of a generic image sensor without the need of a controlled environment is proposed. Starting from a set of heterogeneous CFA images, by using a voting based estimator, the parameters of the noise model are estimated.
\end{abstract}

Keywords: Noise estimation, signal dependent noise, voting-based curve fitting.

\section{INTRODUCTION}

The widespread diffusion of mobile imaging devices and their use in variable light conditions requires accurate estimation of the noise levels, hence image noise estimation is still of paramount importance for effective filtering and image quality. ${ }^{1-3}$

Furthermore, mobile imaging systems are becoming more and more sophisticated with the introduction of features that were before relegated to high-end devices only. Features such as the availability of raw image data for offline processing are being introduced in the market too, hence noise profiling of image sensors maintains its importance for obtaining the best image quality from the imaging pipeline. Filtering noise at the beginning of the imaging pipeline yields improved image quality, however noise profiling of the imager may not be directly available because this data is typically known to the manufacturer only; consequently, it is important to characterize the noise behavior of the image sensor depending on the acquisition conditions. A complete noise characterization typically requires setting up a proper lab configuration using instruments such as integrating spheres, proper light sources, etc. In this paper we propose a methodology based on the analysis of heterogeneous raw images without the need of a properly configured lab for performing the acquisition of test images.

Given the high degree of variability in images, a noise profiling algorithm that works on heterogeneous images must deal with the underlying image signal and structure $;{ }^{4}$ pixels variations related to noise are observable in homogeneous areas only, whereas in areas with high signal activity, noise is masked by the effective image structure. ${ }^{5,6} \mathrm{In}^{7}$ we proposed a solution to estimate the noise profile of an image sensor by using sets of real-world raw images partitioned depending on their acquisition conditions. In this paper we propose a methodology based on a strategy such that the parameters of the noise model are estimated using a voting approach; ${ }^{8}$ this allows reducing the noise samples outliers generated by the misclassification of high signal activity areas that may be erroneously considered as homogeneous. Furthermore, an updated noise modelling equation is also given which yields a better representation of the noise profile for low signal intensities. Noise filters using the updated noise model are able to deliver better results in terms of image structure preservation, avoiding loosing detail in areas of the image in which pixel intensities are low.

The rest of the paper is organized as follows: noise characterization and noise model evaluation are presented in Section 2 and 3 respectively. The proposed noise estimation technique from multiple images is described in Section 4. Finally, experimental results are presented in Section 5 whereas conclusions are summarized in Section 6. 


\section{NOISE CHARACTERIZATION}

The generation of noise during image acquisition depends on several sources (e.g., photon shot noise, read-out noise, thermal noise, amplifiers noise, etc.).

We are interested in characterizing sensor noise in the acquisition phase, hence only the analog gain amplification effects applied at sensor level are considered in our study. For each analog gain, the light intensity is varied by means of an integrating sphere (LE6 of the Image Engineering) such that the acquired images span all the dynamic range of the sensor. For each homogeneous image its average value is computed and is chosen as representative of the intensity $i$; the associated standard deviation is also calculated. We call noise sample the pair $(i, \sigma(i))$, where $i$ is the image average value and $\sigma(i)$ is the standard deviation associated to $i$.

As Fig. 1 shows, once the analog gain factor is set, the noise samples lie in a specific locus that can be identified by a specific equation after a proper fitting process is performed.

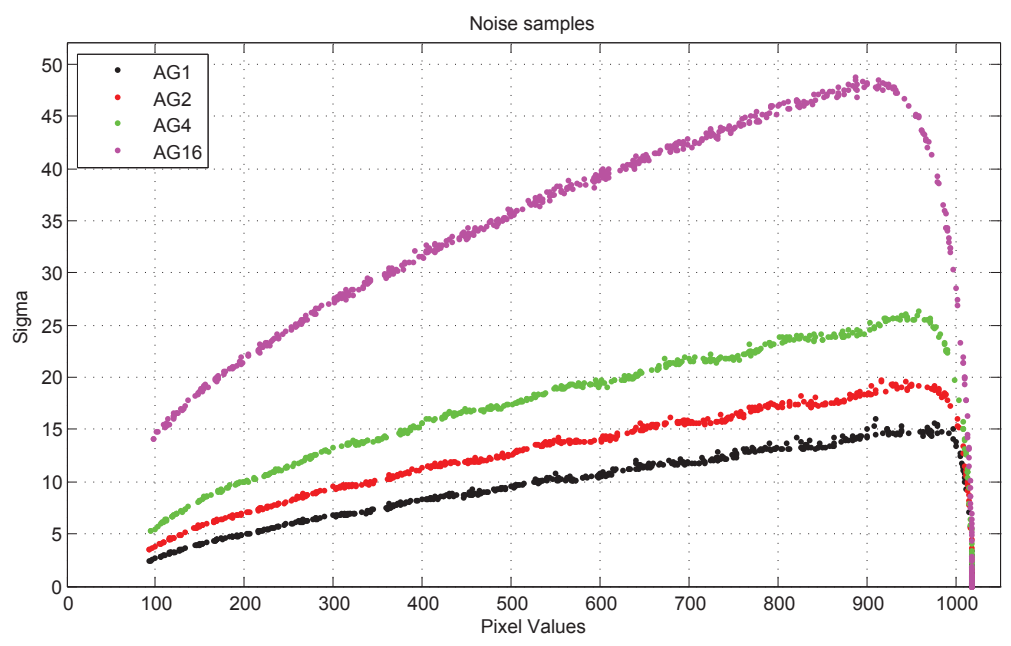

Figure 1. Acquired noise samples at different analog gains $(\mathrm{AG}=1,2,4,16)$.

\section{NOISE MODEL}

As discussed in $^{4}$, the noise samples can be fitted using the signal-dependent model:

$$
\sigma(i)=\sqrt{a \cdot i+b}
$$

where: $a, b \in \mathbb{R}^{+}, i$ is signal intensity.

Hence, we can formulate the problem of characterizing the image sensor noise, as the problem of finding the proper $a$ and $b$ coefficients for any operating condition of the imager (e.g., low light, normal, etc.).

Experiments show that in some cases the fitting curve (1) deviates significantly from the noise samples that we obtained in controlled environment, especially for low signal values (Fig. 2). Notice that the noise samples associated to signal intensities near saturation (clipped values) are excluded from the fitting process.

In order to find an improved fitting equation, we investigated the following alternative functions:

$$
\begin{gathered}
\sigma(i)=a \cdot i^{b}+c \\
\sigma(i)=\frac{a \cdot i+b}{i+c}
\end{gathered}
$$




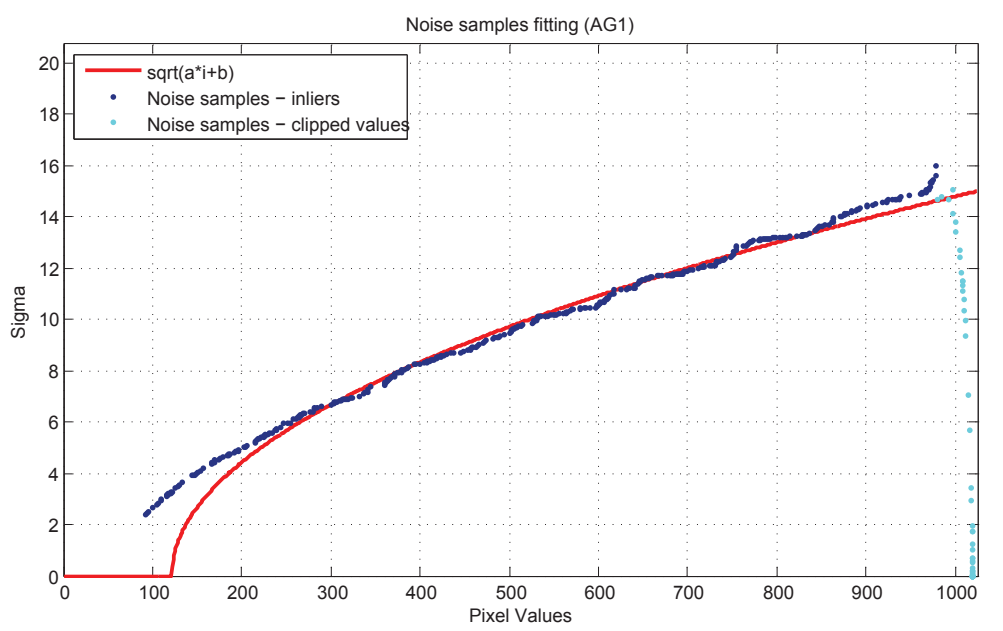

Figure 2. Fitting of noise samples acquired at low analog gain (AG1), using Eq. (1).

Table 1. RMSE comparisons among equations (1), (2), (3) and (4) at different analog gains.

\begin{tabular}{|c||c|c|c|c|}
\hline & Eq. (1) & Eq. (2) & Eq. (3) & Eq. (4) \\
\hline \hline AG1 & 0.4512 & 0.1548 & 0.1801 & 0.1346 \\
AG2 & 0.3291 & 0.1775 & 0.2210 & 0.1634 \\
AG4 & 0.2068 & 0.1853 & 0.2419 & 0.1785 \\
AG16 & 0.1556 & 0.1517 & 0.2184 & 0.1553 \\
\hline \hline average & 0.2857 & 0.1673 & 0.2154 & 0.1579 \\
\hline
\end{tabular}

$$
\sigma(i)=\sqrt{a \cdot i^{2}+b \cdot i+c}
$$

Table 1 and Fig. 3 shows the fitting results and associated RMSE for the analyzed models at different analog gains (AG).

As Table 1 shows, the lowest error is associated to the fitting model (4); this equation allows a more accurate fitting of the noise samples for each considered analog gain especially in the lower part of the dynamic range. By using Eq. (4) the fitting curve gets closer to the noise samples under all analog gains.

\section{NOISE ESTIMATION FROM MULTIPLE IMAGES}

The framework introduced in ${ }^{7}$ allows obtaining the noise profile of a generic image sensor without the need of a controlled environment and specific homogeneous test images; in their place, sets of heterogeneous CFA images partitioned according to their analog gain are processed. A texture detector is used to locate flat areas so that noise statistics can be evaluated in the selected homogeneous areas. We now introduce (4) as a fitting equation and adopt a voting-based approach for finding the proper coefficients for Eq. (4). The characterization process requires finding the appropriate $a, b, c$, coefficients for each available analog gain factor of the sensor.

\subsection{Voting-based Curve Fitting}

Real scenes are usually complex and a texture detector sometimes misclassifies textured areas as homogeneous ones. These errors, including wrong pairs $(i, \sigma(i))$, make the estimation of the parameters of the noise model difficult to obtain with classical curve fitting estimators such as Least Squares. ${ }^{9}$ Estimation methods able to cope with outliers have hence to be considered. ${ }^{10-13}$ Specifically, in this work a robust algorithm based on a voting approach has been employed.

Equation (4), by means of some simple mathematical manipulations, can be rewritten as:

$$
\sigma^{2}(i)=a \cdot i^{2}+b \cdot i+c
$$




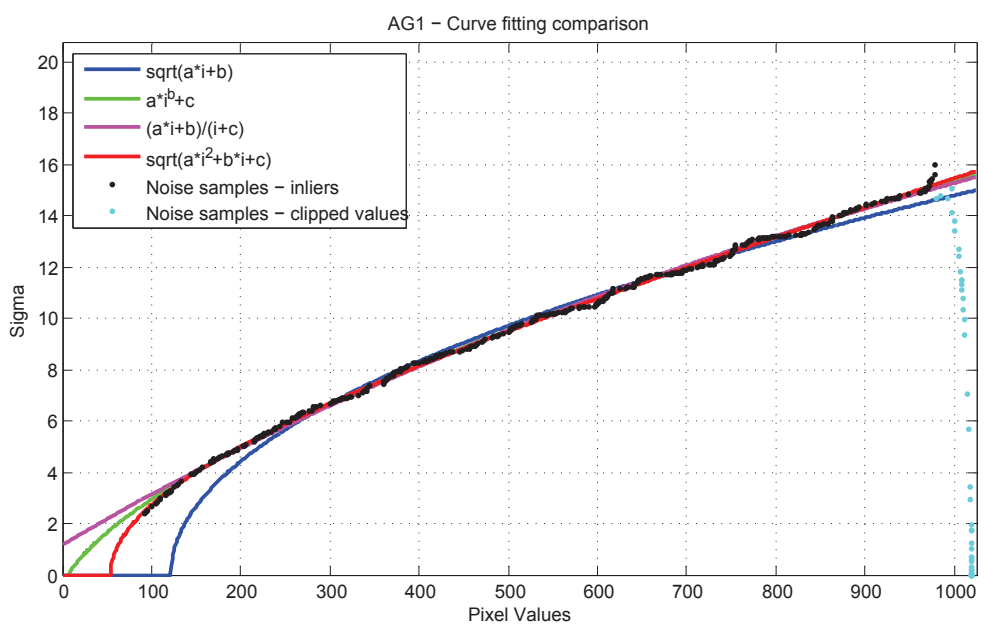

(a)

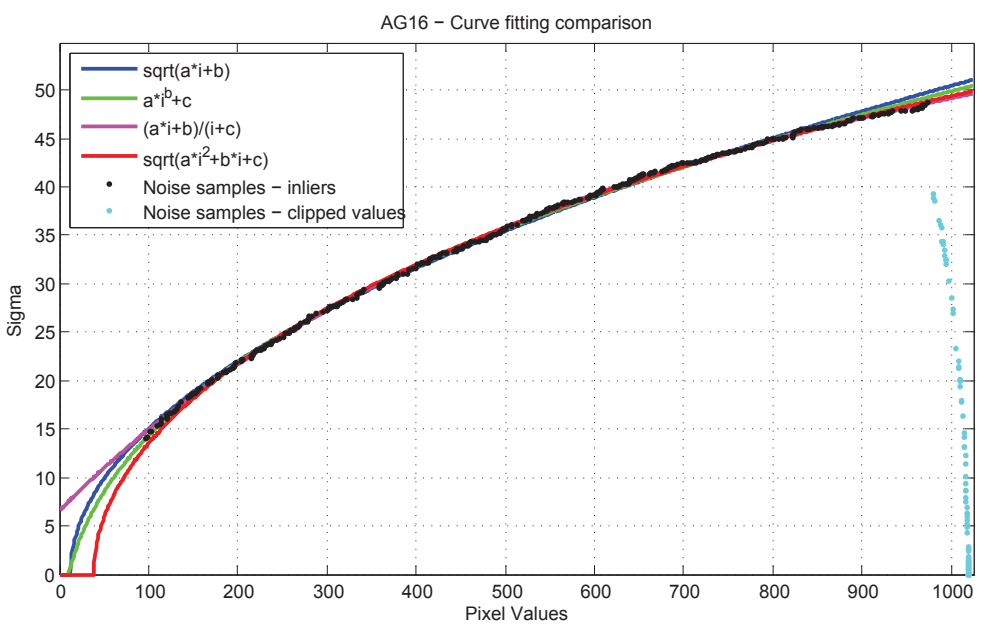

(b)

Figure 3. Fitting noise samples with AG1 $(a)$ and AG16 (b).

Equation (5) represents a plane in the three-dimensional parameter space $(a, b, c)$. Hence, each $(i ; \sigma(i))$ votes for a specific plane in the aforementioned space. The planes related to inliers (i.e., correct pairs) intersect in a small region whereas the ones related to outliers do not disturb this process. An estimation of the coefficients $(a, b, c)$ is then achieved looking for the densest region of the parameter space. A 3D histogram is used to solve this task. As can be easily seen from Fig. 4, the proposed estimator is able to cope with a considerable amount of outliers.

\section{EXPERIMENTS}

The proposed noise profile estimator has been validated using sets of 20 images per each analog gain, acquired using a ST $3 M P$ sensor generating 10bpp raw images. The reported results are obtained using the Kim-Lee texture detector ${ }^{5}$ because of its reliability and reduced outlier amount. The proposed algorithm (voting-based) has been compared with a recent developed technique. ${ }^{7}$ All tests have been performed using the same parameters, a 3D histogram $(a, b, c)$ of $291 \times 12001 \times 11$ bins with $a$ from 0 to $0.0001, b$ from 0.01 to $3, c$ from -60 to 60 . Table 2 shows the improvements in terms of RMSE obtained using Eq. (4) and the voting based fitting method. To visually assess the performances of the proposed system both voting approach $\operatorname{and}^{7}$ are shown in Fig. 5 together with the $(i, \sigma(i))$ pairs measured by the integrating sphere. Estimation error increases as the analog gain augments, but it is possible to reduce the errors by increasing the number of processed images. 


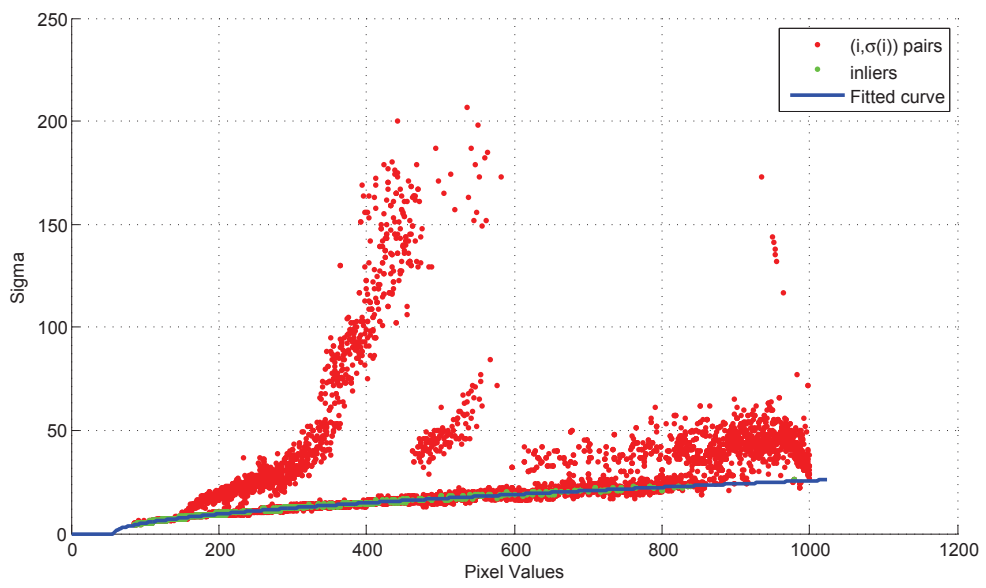

Figure 4. Voting approach selects inliers (green) from the $(i, \sigma(i))$ pair set (red). These inliers are then used to estimate (through Least Squares) the model parameters. The fitting curve is represented in blue.

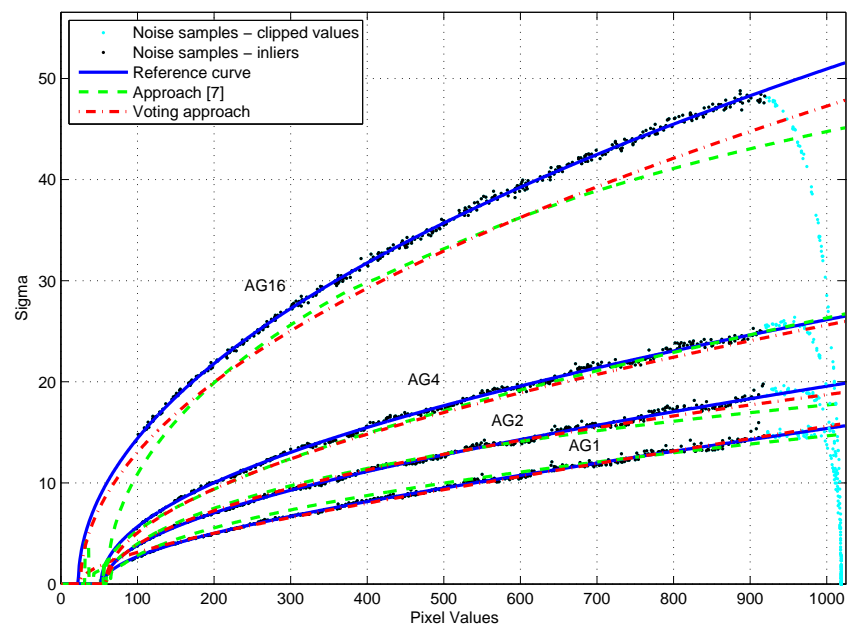

Figure 5. Visual comparison between the voting approach (red) and the method proposed in. ${ }^{7}$ The reference curve (blue) is obtained by fitting the noise samples obtained in lab, using Eq. (4).

\section{CONCLUSIONS}

In this paper the problem of characterizing the sensor signal dependent noise profile also taking into account the analog gain amplification factor has been addressed. Different fitting equations have been tested in order to find the one that minimizes the RMSE associated to the noise samples retrieved in controlled environment. The selected equation has been used with a voting fitting approach and in combination with a texture detector for estimating the noise profile of an imager without the need of homogeneous images. Future works will be devoted to reduce the amount of outliers also in the texture detection step. Moreover, to further evaluate the proposed solution, several sensors will be considered.

Table 2. RMSE comparison between our voting approach and. ${ }^{7}$

\begin{tabular}{|c|c|c|}
\hline & voting approach & 7 \\
\hline \hline AG 1 & 0.2470 & 0.4801 \\
AG 2 & 0.3648 & 0.6463 \\
AG 4 & 0.6981 & 0.5296 \\
AG 16 & 2.7514 & 3.0885 \\
\hline \hline average & $\mathbf{1 . 0 1 5 3}$ & $\mathbf{1 . 1 8 6 1}$ \\
\hline
\end{tabular}




\section{REFERENCES}

[1] Bosco, A., Battiato, S., Bruna, A. R., and Rizzo, R., "Noise reduction for CFA image sensors exploiting HVS behaviour," Sensors 9(3), 1692-1713 (2009).

[2] Bosco, A., Findlater, K., Battiato, S., and Castorina, A., "A noise reduction filter for full-frame data imaging devices," IEEE Transactions on Consumer Electronics 49(3), 676-682 (2003).

[3] Battiato, S., Bruna, A. R., Messina, G., and Puglisi, G., eds., [Image Processing for Embedded Devices], Bentham Science Publisher (2010).

[4] Foi, A., Trimeche, M., Katkovnik, V., and Egiazarian, K., "Practical poissonian-gaussian noise modeling and fitting for single-image raw-data," IEEE Transactions on Image Processing 17(10), 1737-1754 (2008).

[5] Kim, Y.-H. and Lee, J., "Image feature and noise detection based on statistical hypothesis tests and their applications in noise reduction," IEEE Transactions on Consumer Electronics 51(4), 1367-1378 (2005).

[6] Foi, A., Alenius, S., Katkovnik, V., and Egiazarian, K., "Noise measurement for raw-data of digital imaging sensors by automatic segmentation of non-uniform targets," IEEE Sensors Journal 7(10), 1456-1461 (2007).

[7] Bosco, A., Giacalone, D., Battiato, S., and Rizzo, R., "Signal dependent raw image denoising using sensor noise characterization via multiple acquisitions," SPIE Electronic Imaging, Digital Photography VI 7537 (2010).

[8] Shapiro, L. and Stockman, G., [Computer Vision], Prentice-Hall (2001).

[9] Bjorck, A., [Numerical methods for least squares problems], SIAM, Philadelphia, PA (1996).

[10] Fischler, M. A. and Bolles, R. C., "Random sample consensus: a paradigm for model fitting with applications to image analysis and automated cartography," Commun. ACM 24, 381-395 (June 1981).

[11] Hube, P. J., "Robust statistical procedures," SIAM (1996).

[12] Puglisi, G. and Battiato, S., "A robust image alignment algorithm for video stabilization purposes," IEEE Transactions on Circuits and Systems for Video Technology 21(10), 1390-1400 (2011).

[13] Battiato, S., Farinella, G. M., Messina, E., and Puglisi, G., "Robust image alignment for tampering detection," IEEE Transactions on Information Forensics \& Security 7(4) (2012). 